Consumer Price Index), the official Brazilian inflation index, is the main variable (Decree no. 4,937, December 29, 2003). The accumulated CMED escalation amounted to $11.93 \%$ in the studied period, while the purchase prices of cefazolin and ceftriaxone (the antibiotics of choice for surgical prophylaxis and for treatment of pneumonia, urinary tract infections, meningitis, and intraabdominal infections) increased $617 \%$ and $292 \%$, respectively. On the other hand, amikacin and gentamicin, old drugs that are discouraged due to adverse reactions, suffered a $0.9 \%$ reduction and a $3 \%$ increase in the purchase price. Despite the disproportionate increase, the purchase prices did not exceed the maximum prices allowed by CMED. However, the annual trend suggests that the prices charged by laboratories and distributors will be very close to the maximum price allowed for widely marketed hospital antibiotics.

Recently, a small Missouri-based drug maker more than quadrupled the price of nitrofurantoin. ${ }^{5}$ In an interview, the chief executive said he had priced the product according to market dynamics and that it is a moral requirement to make money when you can. Furthermore, he said that this is a capitalist economy, and if you cannot make money, you cannot stay in business.

Clearly, antibiotic prices in Brazil are uncontrolled and antibiotics are in demand from suppliers. Antibiotics are considered the most important drugs in the treatment of serious infections. Many hospitals have avoided the most expensive antibiotics, but this may compromise the treatment of patients. Cost should be part of the
ASP, but it should not be the main engine of an amazing model of therapeutic rationalization.

Acknowledgments. None.

Financial support. No financial support was provided relevant to this article.

Conflicts of interest. All authors report no conflicts of interest relevant to this article.

\section{References}

1. Okumura LM, Silva MM, Veroneze I. Effects of a bundled antimicrobial stewardship program on mortality: a cohort study. Braz J Infect Dis 2015;19: 246-252.

2. Gasparetto J, Tuon FF, Dos Santos Oliveira D, et al. Intravenous-to-oral antibiotic switch therapy: a cross-sectional study in critical care units. BMC Infect Dis 2019;19:650.

3. Schuts EC, Hulscher M, Mouton JW, et al. Current evidence on hospital antimicrobial stewardship objectives: a systematic review and meta-analysis. Lancet Infect Dis 2016;16:847-856.

4. Boyles TH, Whitelaw A, Bamford C, et al. Antibiotic stewardship ward rounds and a dedicated prescription chart reduce antibiotic consumption and pharmacy costs without affecting inpatient mortality or re-admission rates. PLoS One 2013;8:e79747.

5. Crow D. Pharma chief defends $400 \%$ drug price rise as a 'moral requirement.' Financial Times website. https://www.ft.com/content/48b0ce2c-b544-11e8bbc3-ccd7de085ffe. Published September 11, 2018. Accessed April 20, 2020.

\title{
Achieving and maintaining low rates of hospital-onset Clostridioides difficile
}

\author{
Megan Rose Carr LaPorte MD $^{1}$ (D) and Lou Ann Bruno-Murtha DO ${ }^{1,2}$ \\ ${ }^{1}$ Cambridge Health Alliance, Cambridge, Massachusetts and ${ }^{2}$ Harvard Medical School, Boston, Massachusetts
}

To the Editor-The Cambridge Health Alliance (CHA) is a safety-net organization consisting of 277 beds spread between 2 community teaching hospitals and 3 emergency departments. We adopted polymerase chain reaction testing (PCR, Cepheid, Sunnyvale, CA) for Clostridioides difficile infection (CDI) in 2011 and, similar to other hospitals, we noted an increase in our hospital-onset ( $\mathrm{HO}) \mathrm{CDI}$ rate after adopting the more sensitive molecular assay. This increase occurred despite excellent hand hygiene practices, private rooms with contact precautions, daily bleach disinfection of high-touch surfaces, ultraviolet disinfection after terminal cleaning, and an antimicrobial stewardship program. A performance improvement project initiated in 2015 led our organization to successfully reduce the HO-CD standardized infection ratio (SIR) to $<1$ at 2 hospitals. ${ }^{1}$ We have been able to sustain a low HO-CD rate over the past 3.5 years despite changes in the National Healthcare Safety Network's (NHSN's) risk stratification methodology ${ }^{2}$ and without imposing additional testing restrictions on providers. In fact, our SIR has been maintained at $<1$ and has continued to decline.

Author for correspondence: Megan Rose Carr LaPorte, E-mail: mlaporte@cha. harvard.edu.

Cite this article: LaPorte MRC and Bruno-Murtha LA. (2020). Achieving and maintaining low rates of hospital-onset Clostridioides difficile. Infection Control \& Hospital Epidemiology, 41: 995-997, https://doi.org/10.1017/ice.2020.25

\section{Methods}

The initiation of an incentive program in 2015 led our institution to successfully implement a plan in February 2016 to drive the HO-CDI SIR to $<1$ via an automated nurse testing protocol (NTP). The goal of the NTP was to optimize identification of patients with community onset (CO) CDI to avoid inaccurate attribution of HO-CDI and inflation of the SIR due to a delay in stool specimen acquisition. In the NTP, which was embedded in Epic healthcare software (Verona, WI), documentation of diarrhea on the flowsheet during hospital days 1-3 led to a nurse best-practice alert that, when accepted, automatically led to stool collection and PCR testing of the specimen as well as initiation of contact precautions. We were careful to educate nurses and providers that a positive PCR test did not necessarily require antibiotic therapy because PCR detects $C$. difficile bacteria with the gene for toxin production but does not detect the toxin itself. Patients with recent risk factors for CDI or clinical characteristics of disease (eg, fever, severe diarrhea, or leukocytosis) were started on treatment, whereas those who had other reasons for diarrhea (eg, medications, diet, or laxatives) were advised to continue with watchful waiting.

In July 2016, a combined glutamate dehydrogenase (GDH) antigen and toxin assay for toxins A and B (Abbott Diagnostics, 


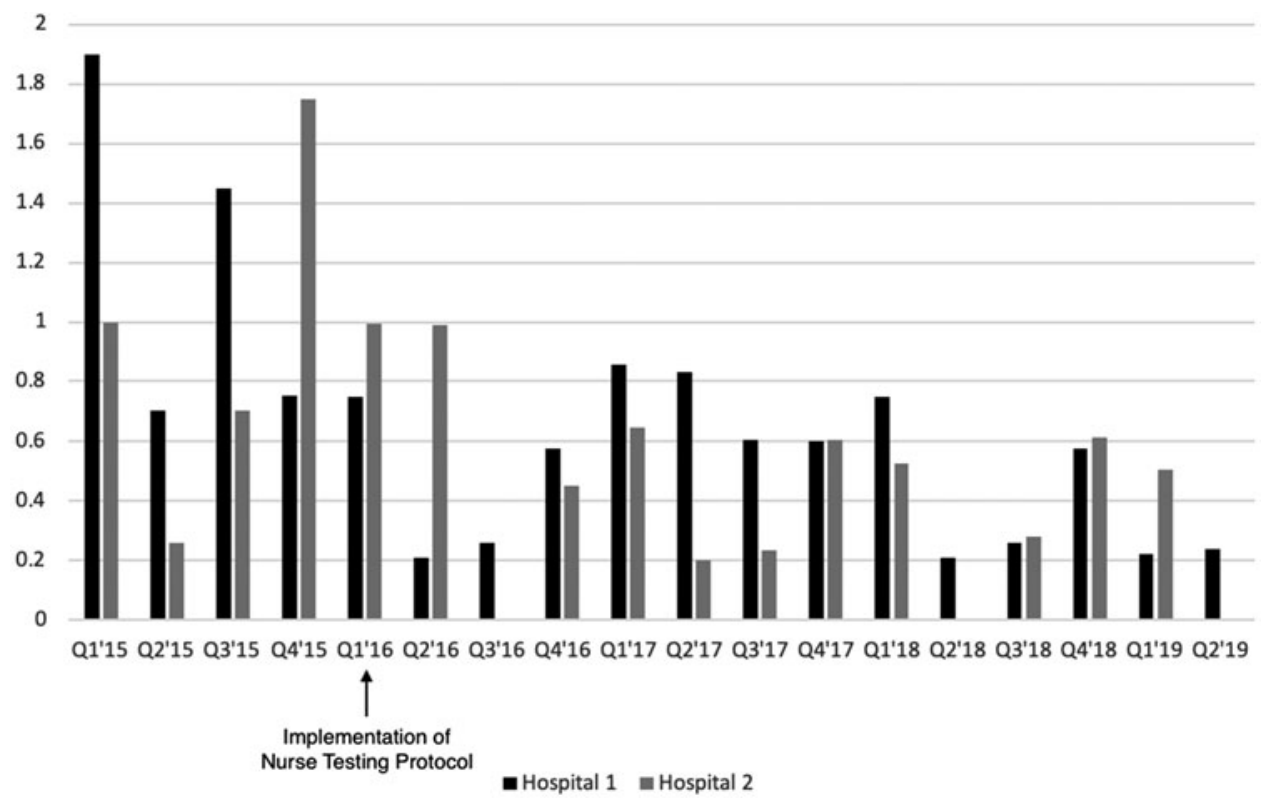

Fig. 1. Clostridioides difficile standardized infection ratio (SIR) over time.
Chicago, IL) was implemented for patients experiencing diarrhea beyond hospital day 3 due to its increased specificity. Diagnostic stewardship measures included limitations of $C$. difficile testing to stool samples taking the shape of the container and 1 test per patient per week.

In January 2018, the NHSN changed its risk stratification according to the last $C$. difficile test performed. ${ }^{3}$ This change had the potential to negatively impact our healthcare-associated CDI metrics. Hence, we report an update on our performance since our prior publication.

\section{Results}

Immediately after implementation of the NTP in February 2016, both CHA sites observed fewer HO-CDI cases than would be predicted according to bed size, hospital affiliation, number of CO-CDI cases, and laboratory test used (Fig. 1). Upon adoption of the GDH/toxin assay in July 2016 and the change in the NHSN risk stratification in January 2018, this improvement was sustained.

\section{Discussion}

Our implementation of an automated process for identifying CO-CDI via the NTP, a process with minimal burden to staff and providers, has driven our CDI SIR to $<1$, and this change has been maintained for nearly 4 years.

Notably, indeterminate GDH and toxin results are reflexed to PCR testing. Although this methodology has the potential to increase our SIR due to the fact that the PCR test is of higher sensitivity, we have maintained this testing strategy (ie, not requiring approval from the infectious disease team to obtain this test) because our excellent performance has been maintained. We argue that delaying PCR testing could lead to delayed diagnosis and treatment of true CDI and therefore pose a danger to patient care.

We also posit that restrictions or hard stops that prevent providers from ordering $C$. difficile testing in the setting of recent laxative use have the potential to delay identification and treatment of CDI. A recent publication supported this argument, ${ }^{4}$ reporting that, despite 2017 IDSA-SHEA guidelines ${ }^{5}$ that recommend against testing for CDI if a patient has received a laxative within the preceding 48 hours, patients who recently received laxatives had no difference in CDI or symptom severity. Had we followed the IDSA-SHEA guidelines, a diagnosis of CDI would have been missed in nearly one-third of the laxative-treated cohort.

Controversy persists regarding the optimal testing strategy for diagnosing CDI. Our approach has been effective in maintaining a low SIR without imposing the need for approval from the infectious disease team or the laboratory, without a hard stop for laxative use in the preceding 48 hours, and without imposing PCR testing restrictions for patients with indeterminate GDH and toxin results. Empowering nurses in both diagnostic and antimicrobial stewardship efforts for CDI continues to be an effective strategy for reducing $\mathrm{HO}-\mathrm{CDI}$.

Acknowledgments. We thank Rebecca A. Osgood, MD, and Casey E. Alexandre, $\mathrm{RN}, \mathrm{BSN}$, for their contributions to this project.

Financial support. No financial support was provided relevant to this article.

Conflicts of interest. All authors report no conflicts of interest relevant to this article.

\section{References}

1. Bruno-Murtha LA, Osgood R, Alexandre C. A successful strategy to decrease hospital-onset Clostridium difficile. Infect Control Hosp Epidemiol 2018;39: 234-236.

2. Surveillance for C. difficile, MRSA, and other drug-resistant infections. Centers for Disease Control and Prevention website. https://www.cdc.gov/ nhsn/acute-care-hospital/cdiff-mrsa/index.html. Published 2019. Accessed December 7, 2019.

3. Multidrug-resistant organism and Clostridioides difficile infection (MDRO/CDI) module. Centers for Disease Control and Prevention website. https:// www.cdc.gov/nhsn/pdfs/pscmanual/12pscmdro_cdadcurrent.pdf. Published 2019. Accessed December 7, 2019. 
4. White NC, Mendo-Lopez R, Papamichael K, et al. Laxative use does not preclude diagnosis or reduce disease severity in Clostridiodes difficile infection. Clin Infect Dis 2019. pii: ciz978. doi: 10.1093/cid/ciz978.
5. McDonald LC, Gerding DN, Johnson S, et al. Clinical practice guidelines for Clostridium difficile infection in adults and children: 2017 update by the Infectious Diseases Society of America (IDSA) and Society for Healthcare Epidemiology of America (SHEA). Clin Infect Dis 2018;66:e1-e48.

\title{
First report of IMP-1 in a clinical isolate of Escherichia coli in Latin America
}

\author{
Priscila Lamb Wink PhD ${ }^{1,2}$, Evelyn Kern Almeida ${ }^{1}$, Marina Niada Crispim ${ }^{1}$, Daiana de Lima-Morales PhD ${ }^{1}$, \\ Alexandre P. Zavascki PhD ${ }^{1,3,4}$ and Afonso Luís Barth $\mathrm{PhD}^{1,2}$ \\ ${ }^{1}$ LABRESIS-Laboratório de Pesquisa em Resistência Bacteriana, Hospital de Clínicas de Porto Alegre, Porto Alegre, RS, Brazil, ${ }^{2}$ Programa de Pós-Graduação em \\ Ciências Farmacêuticas, Faculdade de Farmácia, Universidade Federal do Rio Grande do Sul, Porto Alegre, RS, Brazil, ${ }^{3}$ Department of Internal Medicine, Medical \\ School, Universidade Federal do Rio Grande do Sul, Porto Alegre, Brazil and ${ }^{4}$ Infectious Diseases Service, Hospital de Clínicas de Porto Alegre, Porto Alegre, \\ Brazil
}

To the Editor-The emergence of carbapenem-resistant Enterobacterales (CRE) is a matter of public health concern that seriously compromises antibiotic treatment for severe infections. Since the first report of acquired IMP-1 in Pseudomonas aeruginosa in Japan in $1988,{ }^{1}$ genes encoding IMP enzymes have spread rapidly among Acinetobacter spp and Enterobacterales. ${ }^{2}$ Here, we describe the characteristics of a clinical isolate of $E$. coli harboring $b l a_{\mathrm{IMP}-1}$ gene in Latin America.

An Escherichia coli (termed E. coli 7469F) was recovered from the blood of a patient at Hospital de Clínicas de Porto Alegre in Southern Brazil in May 2019. The E. coli 7469F was not susceptible in vitro to meropenem and ertapenem by the disk-diffusion method. The presence of carbapenemase genes $\left(b l a_{\mathrm{NDM}-1}, b l a_{\mathrm{KPC}-2}, b l a_{\mathrm{VIM}-\mathrm{type}}\right.$, $b l a_{\text {GES-type }}, b l a_{\text {OXA-48-like, }}$ and $\left.b l a_{\mathrm{IMP}-\mathrm{type}}\right)$ was evaluated using multiplex high-resolution melting (HRM) real-time polymerase chain reaction (PCR), ${ }^{3}$ which yielded a positive result only for the $b l a_{\mathrm{IMP}-t y p e}$ gene. The clinical isolate was submitted to conjugation experiment using E. coli $\mathrm{J} 53$ as a receptor, and 1 transconjugant (T7469F) was selected for further analysis. The minimal inhibitory concentrations (MICs) of antibiotics representative of $\beta$-lactams, aminoglycosides, glycilcycline, and chloramphenicol were evaluated by broth microdilution for the E. coli 7469F and its transconjugant (T7469F). The transconjugant $\mathrm{T} 7469 \mathrm{~F}$ presented significant increase in MICs of the carbapenems and ceftazidime compared with E. coli J53 (Table 1). T7469F did not present an increased MIC for aminoglycosides, chloramphenicol, or tigecycline. This whole-genome shotgun project has been deposited at DDBJ/ENA/GenBank under the accession WTVT00000000. The version described here is version WTVT01000000.

The whole genomes of the clinical isolate and its transconjugant were sequenced using the MiSeq platform (Illumina, San Diego, $\mathrm{CA})$, and the data were analyzed using the following tools from the Centre for Genomic Epidemiology website (http://www. genomicepidemiology.org): MLST to characterize sequence typing (ST), ResFinder to characterize antibiotic resistance mechanisms, and PlasmidFinder to characterize plasmid types. Analyses of the

Author for correspondence: Priscila Lamb Wink, E-mail: pris_farma@yahoo.com.br Cite this article: Wink PL, et al. (2020). First report of IMP-1 in a clinical isolate of Escherichia coli in Latin America. Infection Control \& Hospital Epidemiology, 41: 997-998, https://doi.org/10.1017/ice.2020.44

\begin{abstract}
Table 1. Minimal Inhibitory Concentrations (MICs) of Several Antibiotics Used to Treat Escherichia coli 7469F, Transconjugant $7469 \mathrm{~F}$, and E. coli J53
\end{abstract}

\begin{tabular}{lccc} 
& \multicolumn{3}{c}{ MIC (mg/L) } \\
\cline { 2 - 4 } Antibiotics & $\begin{array}{c}\text { E. coli } \\
7469 \mathrm{~F}\end{array}$ & $\begin{array}{c}\text { Transconjugant } \\
\text { T7469F }\end{array}$ & $\begin{array}{c}\text { E. coli } \\
\text { J53 }\end{array}$ \\
\hline Ertapenem & 128 & 8 & $\leq 0.03$ \\
\hline Imipenem & 16 & 4 & 0.5 \\
\hline Meropenem & 64 & 8 & 0.06 \\
\hline Ceftazidime & 512 & 512 & 0.5 \\
\hline Gentamicin & 2 & 2 & 2 \\
\hline Tigecycline & 0.25 & 0.5 & 0.5 \\
\hline Amicacin & 8 & 8 & 4 \\
\hline Chloranphenicol & 8 & 8 & 8 \\
\hline
\end{tabular}

whole-genome sequencing (WGS) data confirmed the presence of the $b l a_{\mathrm{IMP}-1}$ gene in isolate $7469 \mathrm{~F}$ and its transconjugant. Other genes related to resistance to $\beta$-lactam $\left(b l a_{\mathrm{CTX}-\mathrm{M}-15}\right.$ and $\left.b l a_{\mathrm{OXA}-1}\right)$ were found in the clinical isolate using in silico data analyses. E. coli $7469 \mathrm{~F}$ presented 4 plasmids, and the $b \mathrm{I}_{\mathrm{IMP}-1}$ gene was identified in the plasmid IncA/ $C_{2}$. In silico data confirmed that the IncA/C $C_{2}$ was the only plasmid identified in the transconjugant T7469F. Plasmids belonging to the IncA/C incompatibility group are broad host-range vehicles commonly identified among animal and clinical bacterial isolates of Enterobacterales worldwide. This plasmid usually harbors different resistance genes, including $b l a_{\mathrm{CMY}}, b l a_{\mathrm{NDM}}, b l a_{\mathrm{VIM}}$, and bla $_{\mathrm{IMP} .}{ }^{4}$ The WGS analyses also indicated that the E. coli $7469 \mathrm{~F}$ belonged to the ST648. ST648 is a predominant multidrug-resistant ST observed worldwide; it is increasingly reported in multiple regions. $^{5-8}$ In addition, several publications have reported the frequent occurrence of ST648 strains with various $\beta$-lactamases (extended-spectrum $\beta$-lactamases [ESBLs], New Delhi metallo$\beta$-lactamases [NDMs], and Klebsiella pneumoniae carbapenemase [KPCs] $),{ }^{9,10}$ as well as the $m c r-1$ gene. ${ }^{8}$

To the best of our knowledge, this is the first report of a clinical isolate of E. coli ST648 carrying an IncA/C 2 plasmid with the $b l a_{\mathrm{IMP}-1}$ gene in Latin America. Notably, the broad host range 